\title{
An Investigation of Synergy between Batsmen in Opening Partnerships
}

\author{
Juan Valero and Tim B. Swartz \\ Department of Statistics and Actuarial Science, Simon Fraser University, 8888 \\ University Drive, Burnaby British Columbia, Canada V5A1S6 \\ (corresponding author: juancamilo.valero@gmail.com and tim@stat.sfu.ca)
}

\begin{abstract}
In cricket, it has long been accepted that synergies in batting partnerships are an important aspect in batting performance. This paper investigates the importance of partnerships in Test and one-day cricket by comparing the performance of opening batsmen with their "synergistic" partners to the performance of these batsmen with alternative partners. In Test cricket, a bivariate performance statistic based on the strike rate and the batting average is assessed using a nonparametric binomial test procedure. In one-day cricket, a univariate performance statistic based on runs scored relative to resources consumed is assessed using a single sample t-test. Our statistical analyses conclude that synergies in opening partnerships may be considered a sporting myth.
\end{abstract}

Keywords: binomial test, cricket, Duckworth-Lewis, sporting myths, t-test.

\section{Introduction}

Despite the scrutiny that professional sport receives, and the desire for teams and individuals to gain a competitive edge, there exist myths in sport whose consequences are sometimes counterproductive to performance.

The existence of sporting myths was brought to the attention of the general public by Lewis (2003) in his popular book "Moneyball" which was subsequently developed into the 2011 Hollywood blockbuster film starring Brad Pitt. In Moneyball, Billy Beane, the manager of the cash-strapped Oakland A's considered new metrics to uncover value in player evaluation. The Moneyball approach in professional sport has grown and is now prominent in many sports including Major League Baseball (MLB), the National Football League (NFL) and the National Basketball League (NBA).

One reason for the perpetuation of sporting myths may be a consequence of the coaching tradition. Players are often coached by former players who were in turn coached by former players, and so on. Also, the media attention that 
coaches receive may serve as a disincentive to implement radical strategies. For example, a number of academics (see Beaudoin and Swartz, 2010) have unambiguously determined that National Hockey League (NHL) teams should pull their goaltender much earlier (as much as two minutes earlier) when trailing by a goal in the third period. The media criticism that would follow from an increased frequency of empty net goals may be too much for many coaches to handle.

Another example of a sporting myth concerns the hot hand. The hot hand is the widely perceived phenomenon where players experience prolonged periods of exceptional performance and periods of poor performance. In many sports, the hot hand may be viewed as a sporting myth which is explained through natural variation in data (Bar-Eli, Avugos and Raab 2003).

In this paper, we investigate the importance of partnerships in Test and one-day cricket with respect to improved performance. In cricket, batsmen bat in pairs, and the pair is known as a partnership. A partnership continues batting until one of the batsmen is dismissed. It has long been believed that synergies exist between effective partnerships such that the whole is greater than the sum of its parts. A quick Internet search revealed some relevant and colorful quotes:

- Like dancing, in cricket - it takes two to tango ... without a tango/partner - no batsman can singly win a cricket match for his team! (www.itsonlycricket.com/entry/600/)

- India has produced many great Test cricketers who are famous for their partnership building capabilities. (http://cricket-freaks.com/best-partnerships-in-test-odit-cricket-forindia/)

- $\quad$ Test match cricket is about ... scoring runs at a consistent rate. This will be like putting some toothpaste which is there in your hand back into the tube, if there is only one batsman doing this. He needs a partner to be with him holding the other end or scoring alongside him so that the team benefits the most, and stands a greater chance to win Test matches.

(www.cricketdawn.com/cricket-records/test-cricket-records/highestpartnerships-in-test-cricket.html)

Given a bowled ball, the batting action itself is an independent act involving only the striking batsman. Any synergy, if it exists, involves the communication and synchronized running between the wickets by the two batsmen. This sort of symbiosis seems minor compared to the complex interactions between teammates in sports such as basketball and soccer. Consequently, this led us to question the importance of partnerships. In particular, are effective partnerships another example of a sporting myth?

There has been some previous work concerning batting partnerships. Pollard, Benjamin and Reep (1977) endorsed the negative binomial distribution for 
modeling the runs scored for a team in the English County Championships. Croucher (1979) was less satisfied with the adequacy of the negative binomial when applied to 82 Australian partnerships in Australian-England Test matches. Scarf, Shi and Akhtar (2011) also considered the distribution of runs scored in Test cricket partnerships with the aim of addressing batting strategies.

In Section 2, we investigate the effect of partnerships in Test cricket, the version of cricket considered by most to be the traditional form of cricket played at the highest level. In Section 3, we turn our attention to one-day cricket, for which an alternative analysis is required. Some discussion and concluding remarks are then provided in Section 4.

\section{Test Cricket}

Although versions of cricket have been played since the 16th century, Test cricket is considered by most to be the traditional form of cricket played at the highest level. Test cricket matches between two teams may take up to five days to complete where these teams are full member nations of the International Cricket Council (ICC). Currently, there are 10 full members of the ICC and they are Australia, Bangladesh, England, India, New Zealand, Pakistan, South Africa, Sri Lanka, West Indies and Zimbabwe.

To assess the importance of partnerships in Test cricket relative to improved performance, we collected data on Test cricket matches between ICC nations during the period January 2001 through February 2012. In these matches, we recorded the opening partnerships in each innings and we determined the two most common opening partnerships for each team over the time period. In Test cricket matches, there are a maximum of four innings where each team bats twice. By investigating the most common opening partnerships, we attempted to capture cases where batting synergies are believed to exist. In Table 1, the most common opening partnerships are given for 9 of the 10 ICC nations (excluding Zimbabwe). Zimbabwe was excluded from the analysis as they were suspended from Test cricket during the period 2005 through 2011. This resulted in 18 common opening partnerships where the data were collected via the Statsguru search engine at www.espncricinfo.com. In Table 1, we observe that there are three batsmen (V Sehwag, GC Smith, CH Gayle) who are listed twice amongst the most common opening partnerships. This leads to an analysis of 33 different batsmen.

The logic of the data analysis involves a comparison of the performance of batsmen with their common partners (the ones with whom synergies allegedly exist) versus their performance with alternative partners. Should synergies exist, we would expect to see superior performance when batting with common partners. 
To assess batting performance in Test cricket, we note that batsmen attempt to score runs at a high rate relative to balls faced while simultaneously avoiding dismissal. Therefore, we have painstakingly retrieved match summaries and obtained $\left(\mu_{1}, v_{1}\right)$ for each batsman in Table 1 where $\mu_{1}$ is the strike rate (runs scored per 100 balls faced) and, $v_{1}$ is the batting average (runs scored divided by the number of dismissals) where the quantities were calculated over the $m$ innings shared with the common opening partner. Similarly, for each batsman in Table $1,\left(\mu_{2}, v_{2}\right)$ was obtained where the quantities were calculated over innings with alternative partners. It then follows that the bivariate statistic

$$
(x, y)=\left(\mu_{1}-\mu_{2}, v_{1-}, v_{2}\right)
$$

is a measure of comparison of batting with and without the common partner where positive values indicate the presence of synergy.

Table 1: The most common opening partnerships in Test cricket from January 2001 to February 2012 where $m$ denotes the number of innings where the partnership opened together and ni denotes the number of innings where batsman i batted in other partnerships.

\begin{tabular}{|c|c|c|c|c|c|}
\hline \multicolumn{2}{|c|}{ Partnership } & \multirow[b]{2}{*}{ Country } & \multirow[b]{2}{*}{$m$} & \multirow[b]{2}{*}{$n_{1}$} & \multirow[b]{2}{*}{$n_{2}$} \\
\hline Batsman 1 & Batsman 2 & & & & \\
\hline JL Langer & ML Hayden & Australia & 62 & 35 & 75 \\
\hline SM Katich & SR Watson & Australia & 27 & 84 & 44 \\
\hline J Omar & N lqbal T & Bangladesh & 19 & 71 & 13 \\
\hline Iqbal & I Kayes & Bangladesh & 32 & 35 & 11 \\
\hline AJ Strauss & AN Cook & England & 100 & 108 & 79 \\
\hline MP Vaughan & ME Trescothick & England & 54 & 107 & 102 \\
\hline V Sehwag & G Gambir & India & 77 & 98 & 55 \\
\hline V Sehwag & W Jaffer & India & 28 & 98 & 43 \\
\hline L Vincent & MH Richardson & New Zealand & 19 & 26 & 50 \\
\hline JM How & AJ Redmond & New Zealand & 14 & 30 & 5 \\
\hline S Butt & I Farhat & Pakistan & 26 & 47 & 64 \\
\hline M Hafeez & T Umar & Pakistan & 32 & 33 & 56 \\
\hline GC Smith & HH Gibbs & South Africa & 56 & 122 & 91 \\
\hline GC Smith & AB de Villiers & South Africa & 30 & 122 & 102 \\
\hline MS Atapattu & ST Jayasuriya & Sri Lanka & 61 & 45 & 54 \\
\hline TM Dilshan & NT Paranavitana & Sri Lanka & 32 & 96 & 36 \\
\hline CH Gayle & DS Smith & West Indies & 35 & 106 & 42 \\
\hline CH Gayle & D Ganga & West Indies & 47 & 106 & 46 \\
\hline
\end{tabular}

In Figure 1(Appendix), we provide a plot of $(x, y)$ for all 33 batsmen. It appears that the points do not have any obvious pattern that would indicate a presence of synergy. From the plot, the batsman who has the greatest synergy measure is Tamim Iqbal (top right corner). Interestingly, his common partner is Imrul Kayes (bottom right quadrant) who does not exhibit synergy. To assess the presence of synergy more formally, the number of bivariate observations in the first quadrant $W$ is distributed according to the binomial $(33, p)$ distribution. Under the null hypothesis of no synergy, we have $\mathrm{H}_{0}: p=1 / 4$ which we test 
against the alternative hypothesis of synergy $\mathrm{H}_{1}: p>1 / 4$. With $W=10$, the corresponding $p$-value is 0.299 which provides little evidence against $\mathrm{H}_{0}$. We remark that the proposed nonparametric binomial test is appealing since the standard one-way ANOVA procedure relies on normality and the common variance assumption. Also, binomial tests can be carried out for each of the univariate random variables $X$ and $\mathrm{Y}$, and these likewise do not provide evidence of synergy.

Now, it may be argued that the analysis above should be modified so that $\left(\mu_{2}\right.$, $v_{2}$ ) is restricted to only opening partnerships instead of all partnerships not involving the common partner. The restriction results in fewer innings of data and only 30 cases where the batsmen in Table 1 have a direct comparison. The relevant plot is provided in Figure 2(Appendix). In this case, $W=10$ results in a $p$-value of 0.197 which again does not provide evidence against $\mathrm{H}_{0}$.

In conclusion, there does not seem to be any evidence of effective partnerships in Test cricket whereby the whole is greater than the sum of its parts.

\section{One-Day Cricket}

One-day cricket was introduced in the 1960 s as an alternative to traditional forms of cricket. In one-day cricket, there are only two innings of play where each team is allotted a maximum of 50 overs of batting. The restriction on overs requires that matches are completed within a single day. With more aggressive batting, colorful uniforms and fewer matches ending in draws, one-day cricket has become extremely popular. The ultimate event in one-day international (ODI) cricket takes place every four years where the World Cup of Cricket is contested.

We have collected data on all ODI matches involving the 10 full member nations of the ICC during the period January 2002 through February 2012. In these matches, we recorded the opening partnerships in each innings and we determined the two most common opening partnerships for each team over the time period. This resulted in 20 common opening partnerships which are recorded in Table 2. We observe that there are six batsmen ( $\mathrm{T}$ Iqbal, ME Trescothick, V Sehwag, GC Smith, WU Tharanga, CH Gayle) who are listed twice amongst the most common opening partnerships. This leads to an analysis of 34 different batsmen.

In one-day cricket, it is the combination of wickets available and overs remaining in an innings that provides the capacity for scoring runs. The Duckworth-Lewis quantification of the combination of wickets and overs is known as resources (Duckworth and Lewis 1998, 2004). The Duckworth-Lewis method has been accepted as the international standard for the resetting of targets in interrupted one-day matches. The number of resources consumed by 
the batting team during any interval of a match can be determined from the Duckworth-Lewis table.

Following Beaudoin and Swartz (2003), we have used the ratio of total runs scored to total resources consumed as the metric for effective batting in one-day cricket. With this measure, the collection of data for our ODI analysis was a massive undertaking. To appreciate the steps required in the calculation of the ratio, imagine an opening partnership where Batsman A and Batsman B batted together for 15.1 overs (i.e. 15 overs plus one additional ball). In this partnership, Batsman A scored 20 runs in 5.0 overs and Batsman B was dismissed after scoring 30 runs in 10.1 overs. Note that the overs for each batsman were not necessarily consecutive. The Standard Edition of the Duckworth Lewis table indicates that there are:

- $100 \%$ resources available at the beginning of a match

- $82.7 \%$ resources available with 35.0 overs remaining and zero wickets lost

- $78.3 \%$ resources available with 34.5 overs remaining and one wicket lost

Table 2: The most common opening partnerships in ODI cricket from January 2002 to February 2012 where $m$ denotes the number of innings where the partnership opened together and ni denotes the number of innings where batsman $i$ batted in other partnerships

\begin{tabular}{|c|c|c|c|c|c|}
\hline \multicolumn{2}{|c|}{ Partnership } & \multirow[b]{2}{*}{ Country } & \multirow[b]{2}{*}{$m$} & \multirow[b]{2}{*}{$n_{1}$} & \multirow[b]{2}{*}{$n_{2}$} \\
\hline Batsman 1 & Batsman 2 & & & & \\
\hline AC Gilchrist & ML Hayden & Australia & 104 & 81 & 87 \\
\hline BJ Haddin & SR Watson & Australia & 26 & 54 & 102 \\
\hline T lqbal & I Kayes & Bangladesh & 37 & 67 & 28 \\
\hline T lqbal & J Siddique & Bangladesh & 24 & 67 & 29 \\
\hline ME Trescothick & NV Knight & England & 41 & 69 & 30 \\
\hline ME Trescothick & VS Solanki & England & 20 & 69 & 27 \\
\hline V Sehwag & SR Tendulkar & India & 87 & 123 & 136 \\
\hline V Sehwag & SC Ganguly & India & 38 & 123 & 97 \\
\hline NJ Astle & SP Fleming & New Zealand & 34 & 58 & 91 \\
\hline BB McCullum & JD Ryder & New Zealand & 22 & 81 & 21 \\
\hline K Akmal & S Butt & Pakistan & 29 & 84 & 61 \\
\hline I Farhat & M Hafeez & Pakistan & 20 & 34 & 77 \\
\hline GC Smith & HH Gibbs & South Africa & 71 & 117 & 105 \\
\hline GC Smith & HM Amla & South Africa & 34 & 117 & 39 \\
\hline WU Tharanga & TM Dilshan & Sri Lanka & 50 & 79 & 100 \\
\hline WU Tharanga & ST Jayasuriya & Sri Lanka & 49 & 79 & 87 \\
\hline $\mathrm{CH}$ Gayle & S Chanderpaul & West Indies & 39 & 114 & 90 \\
\hline CH Gayle & WW Hinds & West Indies & 40 & 114 & 39 \\
\hline M Masakadza & V Sibanda & Zimbabwe & 25 & 83 & 62 \\
\hline BRM Taylor & S Matsikenyeri & Zimbabwe & 18 & 81 & 76 \\
\hline
\end{tabular}


Accordingly, we assess the match performance of Batsman A by the ratio

$$
20 /\left[5^{*}(100-82.7) /(5+10)\right]=3.47
$$

where we estimate that one third $(5 /(5+10))$ of the resources were consumed by Batsman A based on his proportion of balls faced. Similarly, the match performance of Batsman $\mathrm{B}$ is given by the ratio

$$
30 /[10 *(100-82.7) /(5+10)+(82.7-78.3)]=1.88
$$

where we note the additional chunk of resources consumed by the wicket. Comparing (2) and (3), the proposed metric suggests that Batsman A had a better batting performance than Batsman B in the particular match. However, we emphasize that the performance measures obtained for the ODI batsmen in Table 2 were calculated using the ratio of total runs scored by total resources consumed over all relevant matches. The data collection exercise required the retrieval of match summaries from www.espncricinfo.com and this involved more than 100 hours of investigative work.

In an analysis similar to section 2 , we calculate

$$
x=\mu_{1}-\mu_{2}
$$

where $\mu_{1}$ is the performance metric of a batsman with his common partner and $\mu_{2}$ is the performance metric of the batsman with alternative partners. Positive values of $x$ indicate a presence of synergy in the partnership. Figure 3 (Appendix) provides a dotplot of $x_{1}, \ldots, x_{34}$ in (4) with respect to all of the common opening batsmen in Table 2. The relative balance of negative/positive values of $x$ in the dot plot suggests that there is little evidence of the mystical qualities of partnership. In a one-sided $t$-test of the null hypothesis of no partnership effect using the data $x_{1}, \ldots, x_{34}$, we obtain the $p$-value 0.076 which provides only mild evidence of the existence of effective partnerships. The $q-q$ plot (Figure 4 in Appendix) indicates that the normality assumption is reasonable. To estimate the beneficial effect of common opening partnerships, we calculate the percentage increase in the performance measure due to having a common opening partner relative to the average batting performance. We obtain a very small benefit

$$
\sum x /\left[\sum\left(\mu_{1}+\mu_{2}\right) / 2\right] \rightarrow 4.7 \%
$$

To put the benefit in perspective, an average common batting partnership lasts 14.0 overs and accumulates 36.2 runs. A benefit of $4.7 \%$ confers a paltry $0.047(36.2)=1.7$ runs.

Now, it may be argued that the analysis above should be modified so that $\mu_{2}$ is restricted to only opening partnerships instead of all partnerships not involving the common partner. Due to the restriction, some of the common batsmen have very few innings in opening partnerships with players other than their common partners. We eliminate batsmen from the analysis who have participated in less 
than five innings. This leaves us with 27 cases. The relevant dot plot is provided in Figure 5 (Appendix). In this case, based on the inspection of the dot plot and the $p$-value 0.069 from the one-sided $t$-test, we again conclude that there is only mild evidence of the existence of effective partnerships. A $q-q$ plot (not shown) indicates that the normality assumption is reasonable.

\section{Discussion}

This paper has investigated the longstanding belief in the synergy of partnerships in cricket. The analyzes suggest that the existence of synergies is nothing more than a sporting myth. How might this be explained? Whereas individuals in opening partnerships are often celebrated for their quality, their individual quality is not a consequence of the partnership. These renowned batsmen perform just as well individually when partnered with someone else. Perhaps the general public simply has a better memory for the good events that have taken place in common opening partnerships.

Our analysis of partnerships involved data from both Test cricket and one-day cricket. It would be interesting to see if the same conclusions held true in T20 cricket. A T20 analysis could proceed in the same manner as the one-day analysis where runs scored relative to resources consumed is the natural measure of performance.

Finally, it is interesting to ask whether our findings have any implications for the game itself. It may be the case that teams are reluctant to alter opening partnerships with the fear of destroying something precious and effective. Perhaps our findings may encourage the investigation of optimal batting orders on purely quantitative grounds as described in Swartz et al. (2006).

\section{Acknowledgement}

Swartz has been partially supported by grants from the Natural Sciences and Engineering Research Council of Canada. The authors thank two reviewers whose comments helped improve the manuscript.

\section{References}

1. Bar-Eli, M., Avugos, S. and Raab, M. (2006). Twenty years of "hot hand" research: Review and critique, Psychology of Sport and Exercise, 7: 525553.

2. Beaudoin, D. and Swartz, T.B. (2003). The best batsmen and bowlers in one-day cricket, South African Statistical Journal, 37: 202-222. 
3. Beaudoin, D. and Swartz, T.B. (2010). Strategies for pulling the goalie in hockey, The American Statistician, 64: 197-204.

4. Croucher, S.R. (1979). The battle for the ashes - a statistical analysis of post-war Australia-England cricket, In 4th National Conference of the Australian Society of Operations Research, 48-58.

5. Duckworth, F.C. and Lewis, A.J. (1998). A fair method for resetting the target in one-day cricket matches, Journal of the Operational Research Society, 49: 220-227.

6. Duckworth, F.C. and Lewis, A.J. (2004). A successful operational research intervention in one-day cricket, Journal of the Operational Research Society, 55: 749-759.

7. Lewis, M.M. (2003). Moneyball: The Art of Winning an Unfair Game, W.W. Norton \& Company, Inc, New York.

8. Pollard, R., Benjamin, B. and Reep, C. (1977). Sport and the negative binomial distribution, In Optimal Strategies in Sports (editors S.P. Ladany and R.E. Machol), New York: North-Holland, 188-195.

9. Scarf, P., Shi, X. and Akhtar, S. (2011). On the distribution of runs scored and batting strategy in test cricket, Journal of the Royal Statistical Society: Series A, 174: 471-497.

10. Swartz, T.B., Gill, P.S., Beaudoin, D. and de Silva, B.M. (2006). Optimal batting orders in one-day cricket, Computers and Operations Research, 33: 1939-1950. 


\section{Appendix}

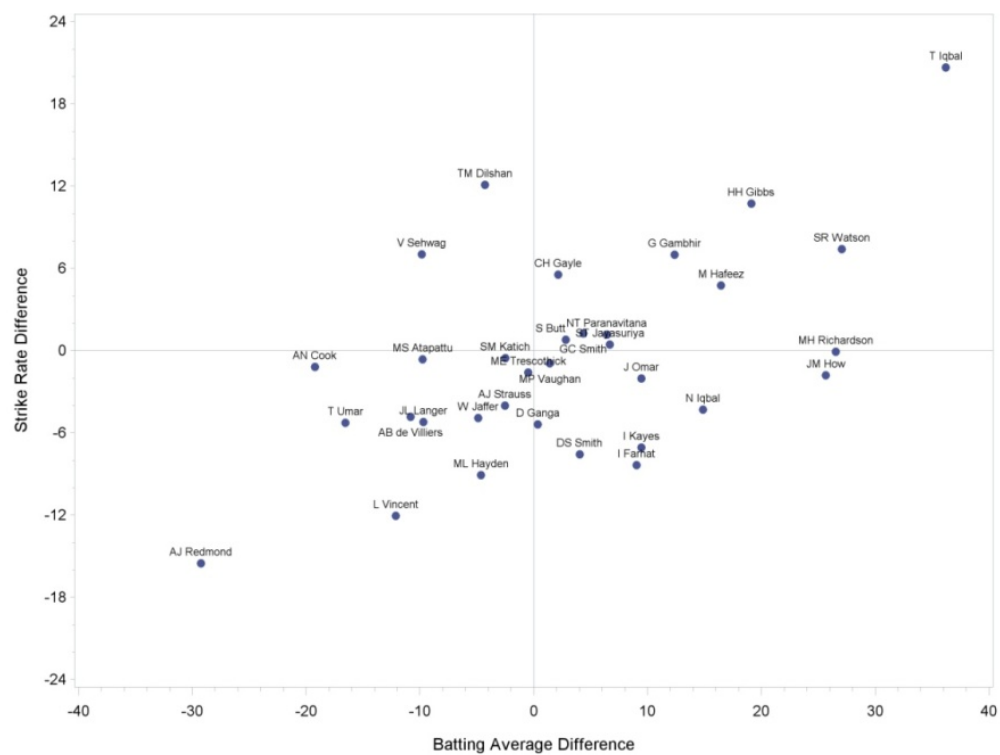

Figure 1: Plot of the 33 points $(x, y)$ in (1) where the comparison involves Test batsmen with their common opening partner versus batsmen with alternative partners.

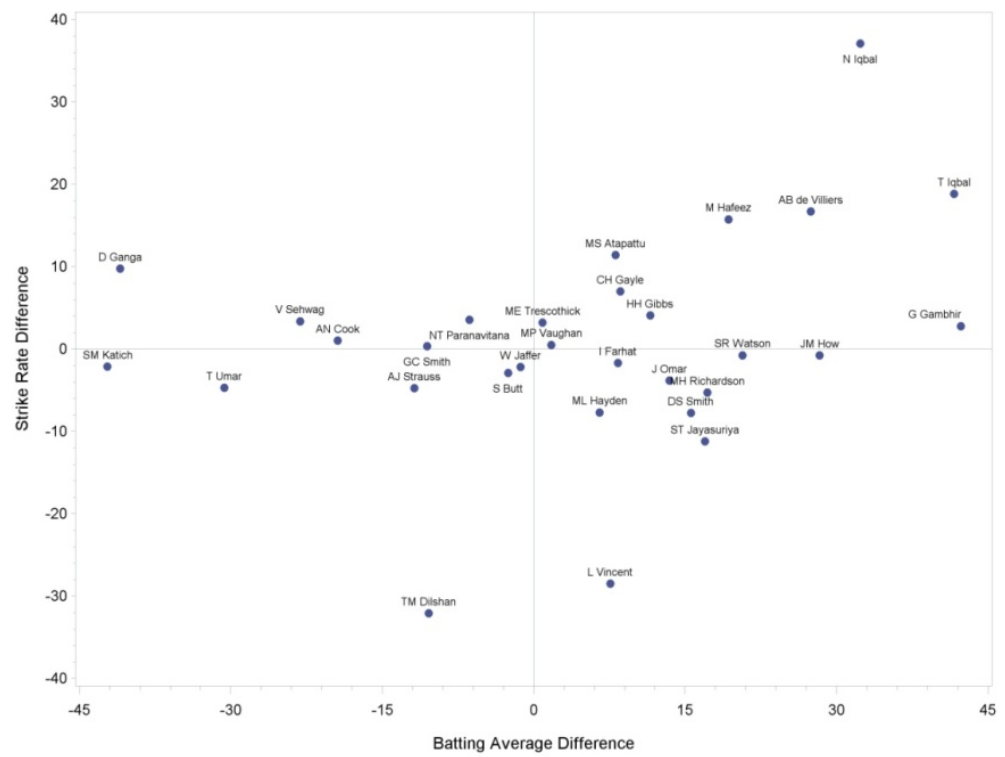

Figure 2: Plot of the 30 points $(x, y)$ in (1) where the comparison involves Test batsmen with their common opening partner versus batsmen with alternative opening partners. 
An Investigation of Synergy between Batsmen in Opening Partnerships

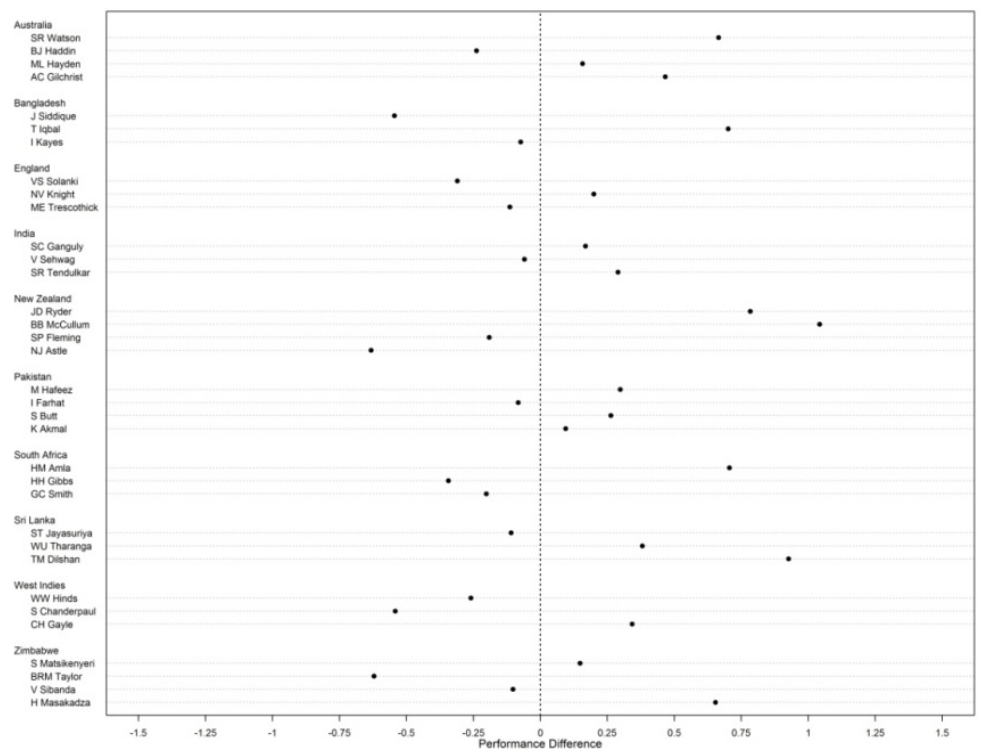

Figure 3: Dot plot of the 34 points $x$ in (4) where the comparison involves ODI batsmen with their common opening partner versus batsmen with alternative opening partners.

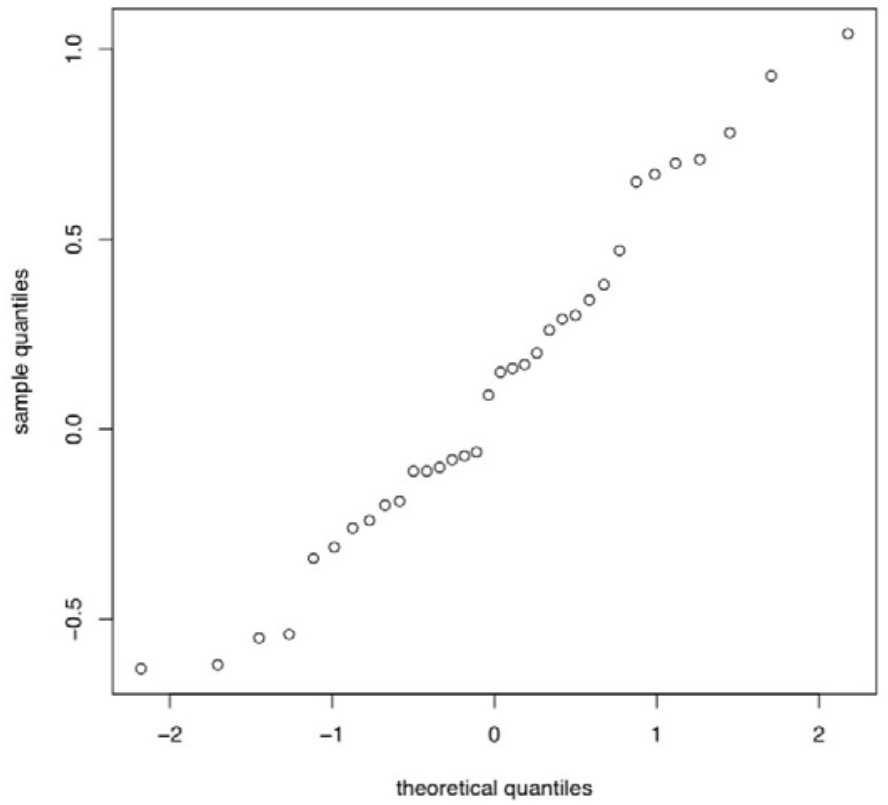

Figure 4: The normal $q-q$ plot corresponding to the 34 points $x$ in (4). 


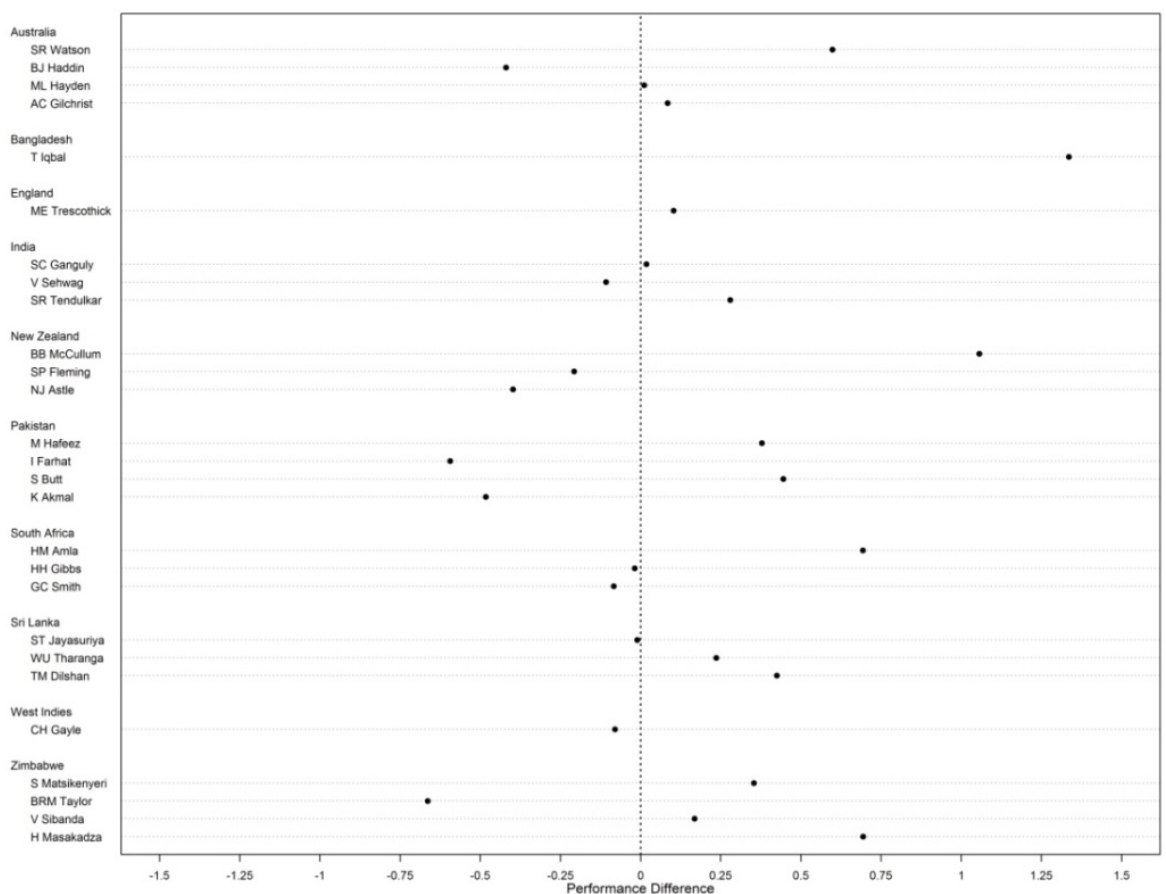

Figure 5: Dot plot of the 27 points $x$ in (4) where the comparison involves ODI batsmen with their common opening partner versus batsmen with alternative opening partners. 